\title{
Largest Atypical Parathyroid Adenoma: A Surgeon's Conundrum
}

\author{
Sarrah Idrees ${ }^{1}$, Manish K Gupta², Rathindra Sarangi ${ }^{3}$
}

\begin{abstract}
Aim: To signify distinguishing features of atypical parathyroid adenoma and parathyroid carcinoma and throw light on immunohistochemistry as a new modality for diagnosis.

Background: Solitary parathyroid adenomas (PAs) are the most common cause of primary hyperparathyroidism (PHPT). Generally, the PAs are small $(<2 \mathrm{~cm})$ and weigh approximately $70 \mathrm{mg}$ to $1 \mathrm{~g}$. Very rarely, some adenomas attain large sizes of $>2 \mathrm{~cm}$ or weigh $>2 \mathrm{~g}$. These are termed as "Giant parathyroid adenoma (GPA)." Some of these may have few histological characteristics of parathyroid carcinoma (PC) but not sufficient to be diagnosed as cancer. These are categorized as atypical adenomas. We report a case of the largest atypical parathyroid adenoma.

Case description: A 47-year-old gentleman, diagnosed with fracture shaft of the right femur, was found to have hypercalcemia (S. Cal 13.6) on routine biochemical examination along with deranged calcium metabolic profile and osteoporosis on bone scan. SestaMIBI scan and MRI reported a large PA in relation to right lobe of thyroid. Intraoperatively, a large adenoma measuring $6 \times 4 \times 3 \mathrm{~cm}$ was excised along with fracture fixation. Histopathology reported it as atypical parathyroid adenoma. Postoperatively, he developed hungry bone syndrome, which required intravenous and oral calcium and vitamin D supplementation. He became euglycemia 2 months after surgery.

Conclusion: Atypical giant PA is a rare entity. Molecular phenotype of atypical adenomas are p27+, bcl+, ki67-, mdm+ but none of these is positive in carcinoma. Histopathology is a must for distinguishing these two, but this also may fail in tumors with equivocal features. Therefore, the paradigm for the diagnosis of these entities has shifted to immunohistochemistry, which holds a promising future.

Clinical significance: Differentiating these two conditions is imperative in order to manage both and to keep patients of atypical parathyroid adenoma in close follow up.

Keywords: Atypical parathyroid adenoma, Giant parathyroid adenoma, Parathyroid carcinoma.

World Journal of Endocrine Surgery (2019): 10.5005/jp-journals-10002-1257
\end{abstract}

\section{INTRODUCTION}

Primary hyperparathyroidism (PHPT) is the third most common endocrine disorder after diabetes mellitus and hypothyroidism. ${ }^{1}$ Solitary PAs constitute $85 \%$ of all the cases of PHPT, whereas PCs constitute only $<1 \% .^{2}$ Generally, the PAs are small $(<2 \mathrm{~cm})$ and weigh approximately $70 \mathrm{mg}$ to $1 \mathrm{~g}^{3}$ Very rarely, some adenomas attain large size of $>2 \mathrm{~cm}$ or weigh $>2 \mathrm{~g}$. These are termed as "Giant parathyroid adenoma (GPA)." Furthermore, some adenomas may have few histologic characteristics of PC but not sufficient to be diagnosed as cancer. These tumors are categorized as atypical adenomas. ${ }^{4}$ Only a few giant atypical adenomas have been published in literature, and we report a case of the largest atypical parathyroid adenoma until date.

\section{Case Description}

A gentleman, aged 47 years, presented to our emergency department with complaints of severe pain and swelling in the right thigh for 1 day following trivial trauma. He also gave a history of generalized body ache and bony pains for the past 1 year but with no other significant symptoms. The orthopedics team evaluated him, and a diagnosis of fracture shaft of right femur was made (Fig. 1). He was planned for an open reduction and internal fixation. On routine biochemical examination, hypercalcemia was detected (S. Cal 13.6). Our surgical team was then called for and owing to the hypercalcemia; a complete calcium metabolic profile was done. His serum parathyroid hormone was $1,900 \mathrm{pg} / \mathrm{mL}$, serum vitamin D3 was $4 \mathrm{mg} / \mathrm{dL}$, serum phosphorous was $2 \mathrm{mg} / \mathrm{dL}$, serum alkaline phosphatase was $536 \mathrm{IU} / \mathrm{L}$, serum magnesium was $1.3 \mathrm{mg} / \mathrm{dL}$ and
${ }^{1-3}$ Department of General and Laparoscopic Surgery, Sir Ganga Ram Hospital, New Delhi, India

Corresponding Author: Sarrah Idrees, Department of General and Laparoscopic Surgery, Sir Ganga Ram Hospital, New Delhi, India, Phone: +91 9561575999, e-mail: sid.rebelious@gmail.com

How to cite this article: Idrees S, Gupta MK, et al. Largest Atypical Parathyroid Adenoma: A Surgeon's Conundrum. World J Endoc Surg 2019;11(2):53-56.

Source of support: Nil

Conflict of interest: None

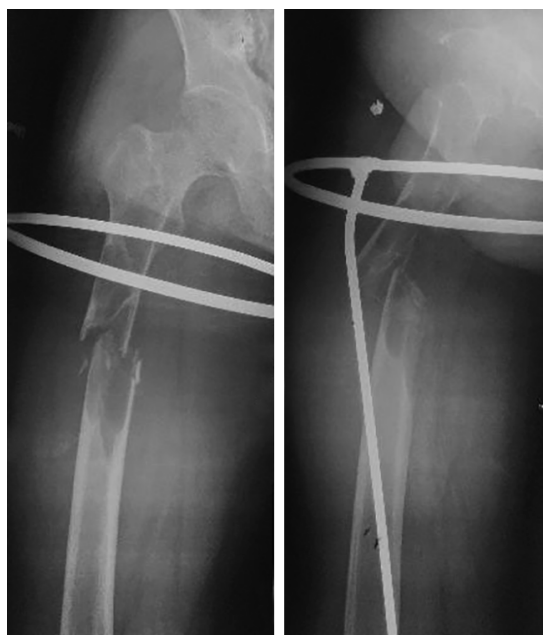

Fig. 1: X-ray AP view shaft of femur showing fracture

(-) The Author(s). 2019 Open Access This article is distributed under the terms of the Creative Commons Attribution 4.0International License (https://creativecommons. org/licenses/by-nc/4.0/), which permits unrestricted use, distribution, and non-commercial reproduction in any medium, provided you give appropriate credit to the original author(s) and the source, provide a link to the Creative Commons license, and indicate if changes were made. The Creative Commons Public Domain Dedication waiver (http://creativecommons.org/publicdomain/zero/1.0/) applies to the data made available in this article, unless otherwise stated. 
serum albumin was $3.68 \mathrm{~g} / \mathrm{dL}$. Two-site bone mineral density scans (DEXA-BMD) reported osteoporosis with severely increased fracture risk as compared to average peak BMD in healthy young patients. The $T$-score of the lumbar and hip region was -2.6 and -1.8 , respectively, and the $Z$-score was -3.4 and -2.8 , respectively. SestaMIBI scan confirmed PHPT due to a large PA in relation to the right lobe of thyroid gland. MRI neck reported an ill-defined hypointense soft tissue lesion of size $6 \times 4 \times 3 \mathrm{~cm}$ in relation to the right lobe of soft tissue lesion thyroid compressing the trachea laterally (Figs 2 and 3). Decision for parathyroidectomy with internal fixation of shaft of femur was taken in consultation with orthopedics team after complete workup and informed consent from patient and his relatives.

The patient was planned for excision of the PA first, after optimum preoperative hydration. Intraoperatively, a large, firm adenoma measuring $6 \times 4 \times 3 \mathrm{~cm}$ was noted in relation to the lower pole of the right thyroid lobe and the carotid sheath laterally. It could be easily separated from the surrounding structures. Nailing and plating of fracture shaft of the femur was also done in the same setting by the orthopedics team.

The specimen was sent for histopathological examination. On gross examination, the right inferior parathyroid gland was noted to be completely encapsulated with smooth outer surface measuring $6 \times 4 \times 3 \mathrm{~cm}$ and weighing $80 \mathrm{~g}$. The anterior surface was solid, cystic with two cysts measuring 0.5 and $0.8 \mathrm{~cm}$ in diameter. These were filled with hemorrhagic fluid. The rest of the solid surface was gray-brown with areas of congestion. Microscopically, well-circumscribed and thinly encapsulated tumors composed of closely packed groups, and nests of cells separated by vascular fibrous septa were observed. The cells were polyhedral with monomorphic nuclei with minimal pleomorphism. In an area, thick fibro-collagenous septa intersecting the tumor cells were noted.
Many acini showed cystic dilatation with proteinaceous material in their lumina. Focal pseudocapsular invasion was also noted. No capsular or vascular invasion, increased mitosis or necrosis was observed. The large size of the adenoma, increased weight, thick fibrous septae, pseudocapsular invasion, increased parathyroid hormone (PTH), and serum calcium levels favored a diagnosis of atypical parathyroid adenoma (Fig. 4).

Intact PTH sent 10 minutes after PA excision was reported to be $195 \mathrm{pg} / \mathrm{mL}$. The patient developed severe hypocalcemia on postoperative day 1 (POD) (serum calcium-6.03 mg/dL) which required intravenous (IV) calcium gluconate supplementation 8 hourly along with oral calcium supplementation $2 \mathrm{~g} 12$ hourly and orally active vitamin D $0.25 \mu \mathrm{g} 12$ hourly. The IV calcium requirement reduced to once daily on POD4 (serum calcium $7.39 \mathrm{mg} / \mathrm{dL}$ ) and symptoms of hypocalcemia subsided. He was discharged on POD7 (serum calcium of $7.4 \mathrm{mg} / \mathrm{dL}$ ) on oral calcium $1 \mathrm{~g} 6$ hourly and vitamin $D 0.25 \mu \mathrm{g} 12$ hourly.

On the first follow-up after 1 week, the calcium dose was reduced to $1,000 \mathrm{mg} 8$ hourly in view of serum calcium being 8 $\mathrm{mg} / \mathrm{dL}$. One month after the surgery, serum calcium was $8.94 \mathrm{mg} /$ $\mathrm{dL}$, and so the dose was further reduced to $1 \mathrm{~g} 12$ hourly and oral vitamin D once daily. The dosage of calcium was titrated at regular intervals, and finally, 2 months after the surgery, the patient became euglycemia $(9.2 \mathrm{mg} / \mathrm{dL})$ and was weaned off the medications. The fracture shaft of the right femur has also healed well.

\section{Discussion}

PHPT is associated with reduced bone mineral density, mainly at sites rich in cortical bone (femur, radius, and spine). Even though femur is the longest, strongest, and heaviest bone in our body, it is still prone to fracture because it constitutes a part of the principal load-bearing skeleton. Therefore, in a previously osteopenic/osteoporotic bone,
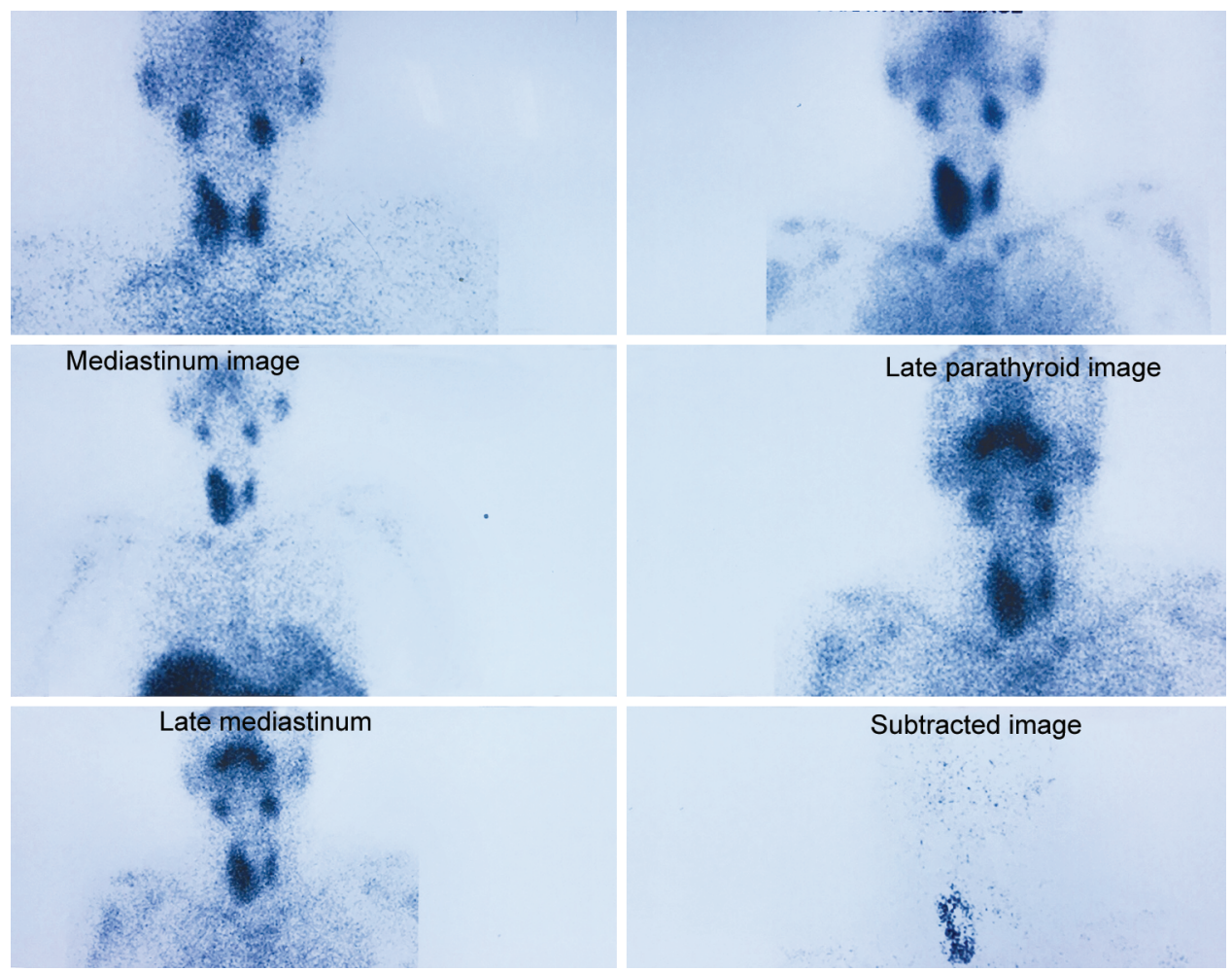

Fig. 2: SestaMIBI scan depicting an enlarged right parathyroid gland 

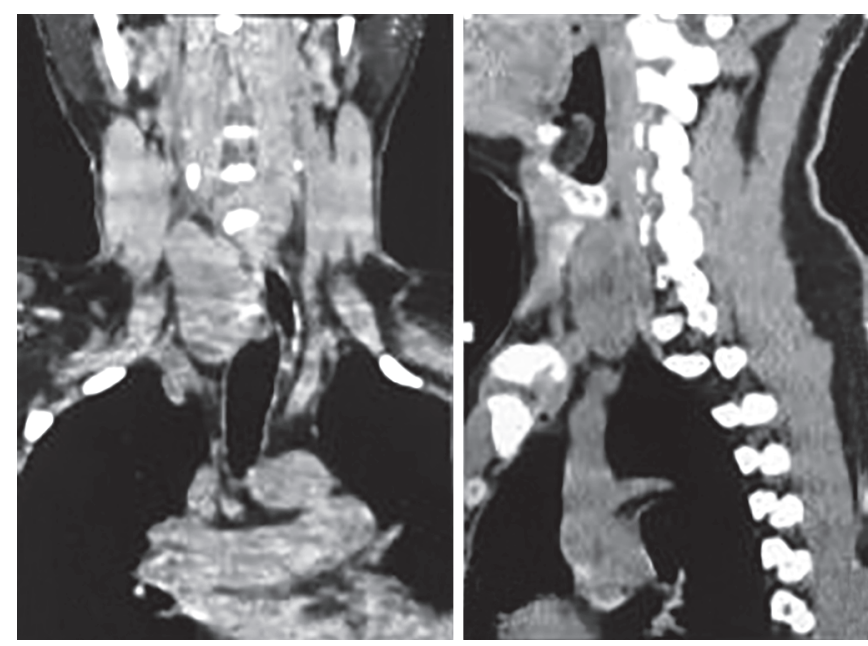

Fig. 3: MRI neck (coronal and sagittal sections showing well-defined hypo intense lesion in relation to the inferior pole of right lobe of thyroid

as is in PHPT, even low energy falls can cause fracture. Surgery is the mainstay in the management of femur fractures because of the high rate of union, low rate of complications, and the advantage of early fracture stabilization. Conservative management of femur fracture is suggested only in children or adults who are unfit for surgery. As per the fourth international guidelines, for management of PHPT, vertebral imaging or assessment of fracture risk by dual-energy X-ray absorptiometry scan (DEXA) is recommended to exclude asymptomatic vertebral fracture and assess bone health.

Atypical giant PA is a rare entity. Their overall characteristics are intermediate between typical PA and PC. Demographically, atypical PAs are significantly more in men. PCs are also more common in men, but females have a higher risk of developing typical PAs. ${ }^{4}$ The range of S. Ca levels has been found to be highest in PC followed by atypical PA and then typical PA. ${ }^{4}$ No significant difference has been noted in the S. PTH levels in these conditions. However, a significant point to note is that GPAs are more frequently asymptomatic as compared to non-GPAs despite higher levels of calcium. ${ }^{1}$ Another clinical difference reported was that PC presented with a palpable neck mass in about one-third of patients, whereas none or extremely rarely do GPA present with this finding.

Radiologically, the depth width ratio larger or smaller than one is the most discriminatory ultrasonography parameter to differentiate between GPA and PC. ${ }^{1}$ It is larger than 1 in $95 \%$ of PCs and smaller than 1 in $95 \%$ of GPAs. Both are presented as hypoechoic masses radiographically, but PCs are more hypoechoic and have a more lobulated and heterogeneous appearance. On the contrary, GPAs have smooth margins and are homogenous. On color Doppler, PCs are vascular and typically show a peripheral vascular arc and prominent polar feeding vessel arising from the branches of inferior thyroidal artery. Atypical features such as cystic degeneration, calcification, and greater infiltration into surrounding tissues all favor a carcinomatous lesion. It is unadvisable to attempt to distinguish between benign and malignant parathyroid lesions by fineneedle aspiration cytology (FNAC) because of the following reasons: (a) FNAC has low discriminating capacity, (b) risk of tumor dissemination with FNAC is high. ${ }^{4}$

It is equally difficult to make a likely diagnosis of PC or atypical adenoma intraoperatively. PCs have been observed to be large, hard, surrounded by fibrous tissue and are densely adherent to surrounding structures. Atypical adenomas also tend to have similar

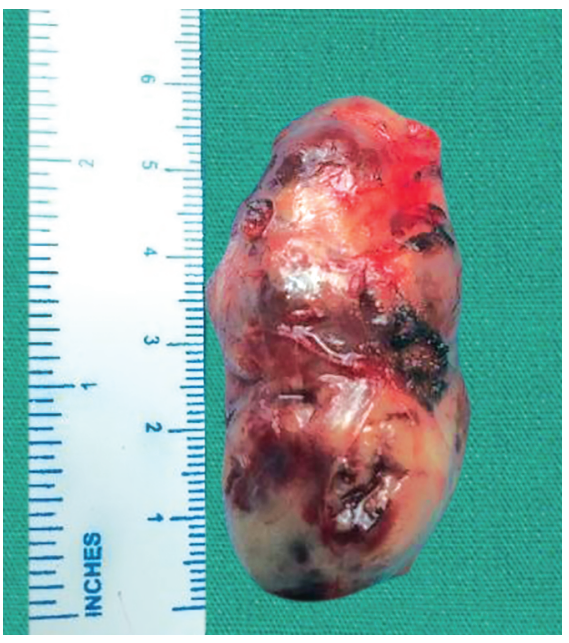

Fig. 4: Gross specimen of giant atypical parathyroid adenoma excised

characteristics except that the adherence to surrounding structures may be less. ${ }^{4}$ In case of any doubt, frozen section of the en-bloc resected specimen may be sent. The only means of differentiating $P C$ with an atypical adenoma is via microscopic examination. Both are rare histopathologic entities.

The presence of two or more of the following point to the diagnosis of PC; (a) incomplete invasion of capsule, (b) fibrous bands, (c) exaggerated trabecular growth, (d) mitotic activity $>1 / 10$ hpf, (e) tumor necrosis with capsular or vascular invasion. The presence of mitosis should be investigated to exclude invasive growth. ${ }^{2}$ Hence, the tumors with corresponding features, but not diagnostic of malignancy, fall under the umbrella of "atypical adenoma." Molecular phenotype of atypical adenomas is again intermediate between typical adenomas and carcinoma. p27+, bcl+, ki67, mdm+ were noted in $76 \%$ and $29 \%$ of typical and atypical adenomas, respectively, but none in carcinoma. ${ }^{2}$ Also, a main genetic alteration (HRPT2 gene mutation) is responsible for PC. Parafibromin staining is therefore negative in PCs, whereas in atypical adenomas, no genetic alteration is present and parafibromin staining is positive. The largest atypical PA reported by Ramaswamy et al. measured $5 \times 3.5 \times 1 \mathrm{~cm}$.

In our case, the adenoma not only measured $6 \times 5 \times 4 \mathrm{~cm}$ and weighed $80 \mathrm{~g}$ but was also reported to be an atypical adenoma. This is the largest atypical PA to be reported in literature. (To the best of our knowledge as per our search of published data.)

\section{Conclusion}

Atypical PA and PC occupy different niches in the spectrum of PHPT. Both differ vastly in their natural course of disease and management options. Histopathology is imperative for distinguishing atypical adenoma from carcinoma, but this too may fail to do so in tumors with equivocal features. ${ }^{5}$ Therefore, the paradigm for the diagnosis of these entities has now shifted to immunohistochemistry, which holds a promising future in differentiating atypical PA and PC.

\section{References}

1. Araujo Castro M, López AA, et al. Giant parathyroid adenoma: differential aspects compared to parathyroid carcinoma. Endocrinol Diabetes Metab Case Rep 2017;2017:17-0041. DOI: 10.1530/EDM-170041. 
2. Ramaswamy AS, Vijitha T, et al. Atypical parathyroid adenoma. Indian J Pathol Microbiol 2017;60:99-101. DOI: 10.4103/0377-4929. 200055.

3. Jayant M, Kaushik R, et al. Giant Parathyroid Adenoma. Kathmandu Univ Med J 2011;33(1):77-79. DOI: 10.3126/kumj.v9i1.6269.
4. Fernandez-Ranvier GG, Khanafshar E, et al. Parathyroid carcinoma, atypical parathyroid adenoma, or parathyromatosis? Cancer 2007 Jul 15;110(2):255-264. DOI: 10.1002/cncr.22790.

5. Wang J, Murugan $P$, et al. Browntumours and the atypical parathyroid adenoma. AACE Clin Case Rep Summer 2017;3(3):233-238. 